\title{
L'histoire inachevée de la régulation du marché du riz à Madagascar
}

The ongoing story of rice market regulation in Madagascar

Marie-Hélène Dabat, Olivier Jenn-Treyer, Simon Razafimandimby et Louis Bockel

\section{(2) OpenEdition}

\section{Journals}

Édition électronique

URL : http://journals.openedition.org/economierurale/535

DOI : 10.4000/economierurale.535

ISSN : 2105-2581

Éditeur

Société Française d'Économie Rurale (SFER)

Édition imprimée

Date de publication : 1 juin 2008

Pagination : 75-89

ISSN : 0013-0559

Référence électronique

Marie-Hélène Dabat, Olivier Jenn-Treyer, Simon Razafımandimby et Louis Bockel, « L'histoire inachevée de la régulation du marché du riz à Madagascar », Économie rurale [En ligne], 303-304-305 | Janvier-juin 2008, mis en ligne le 01 juillet 2010, consulté le 21 décembre 2020. URL : http:// journals.openedition.org/economierurale/535; DOI : https://doi.org/10.4000/economierurale.535 


\title{
L'histoire inachevée de la régulation du marché du riz à Madagascar
}

\author{
Marie-Hélène DABAT • CIRAD, URP Système de culture et rizicultures durables (SCRiD), Antananarivo, \\ Madagascar, CIRAD, URP SCRID, Montpellier
}

Olivier JENN-TREYER • Secrétariat Multi-Bailleurs, Antananarivo

Simon RAZAFIMANDIMBY • Centre national de recherche appliquée au développement rural (FOFIFA), URP SCRID, Antsirabe

Louis BOCKEL •FAO, Agricultural Policy Support Service, Rome

$\dot{A}$ Madagascar, le riz est au cœur des vies économique, sociale et politique depuis l'unification nationale dont le principal initiateur, le souverain Andrianampoinimerina (1786-1810), avait déjà comme programme politique principal : «la mer sera la limite de ma rizière ». Depuis cette époque, l'histoire du secteur rizicole et de sa régulation se caractérise par un perpétuel mouvement de balancier entre l'intervention de l'État et des tentatives de pilotage par le marché, entre la défense d'un prix acceptable par le consommateur et le soutien des prix aux producteurs.

La filière riz malgache est structurellement peu performante dans le sens où elle donne lieu à une accumulation limitée du capital. Le regard que nous nous proposons de porter ici est une tentative d'explication de la faible performance de la filière et une analyse de ses modes de régulation au cours de plusieurs décennies jusqu'à une situation de crise récente qui a stigmatisé l'intervention directe de l'État sur le marché et dont l'enjeu est la création de nouvelles règles entre acteurs pour relancer la croissance de la filière. Le cadre théorique sur lequel nous avons choisi de nous appuyer est celui de la nouvelle économie institutionnelle (Williamson, Kydd et Dorward, North, Hoff). Il nous servira à comprendre l'incapacité de la filière riz à accumuler durablement le capital et à expliquer en quoi de nouveaux outils dont se dote la filière pourraient créer plus de coordination entre les acteurs.
Les mécanismes d'un nouveau mode de régulation sectoriel, qui à la fois favorise l'expansion économique, évite les chocs sociaux et préserve les capacités de choix des acteurs, restent à définir. L'apparition récente d'un nouvel outil de pilotage de la filière, fruit d'un arrangement institutionnel public/privé et matérialisé par la mise en place d'une plateforme de concertation entre acteurs, pourrait y contribuer. Cette forme de coordination hors-marché, à la recherche de compromis institutionnalisés, pourrait favoriser des choix économiques et sociaux équilibrés favorables à une recomposition durable de la filière et du marché rizicoles.

Les enjeux de durabilité associés à la production de riz à Madagascar seront d'abord exprimés, ensuite nous tenterons une explication institutionnelle des faibles performances économiques de la filière riz, puis l'évolution historique des modes de régulation du marché rizicole depuis les années soixante jusqu'à la crise de 2004 sera retracée, enfin il conviendra de s'interroger sur les modes de régulation les plus à même d'engendrer la croissance durable de la filière riz.

\section{Un véritable enjeu de développement durable}

L'importance du riz à Madagascar en fait un secteur privilégié pour atteindre les objectifs économiques, sociaux et écologiques du développement durable. Cette filière a un impact inégalé sur la croissance économique, 


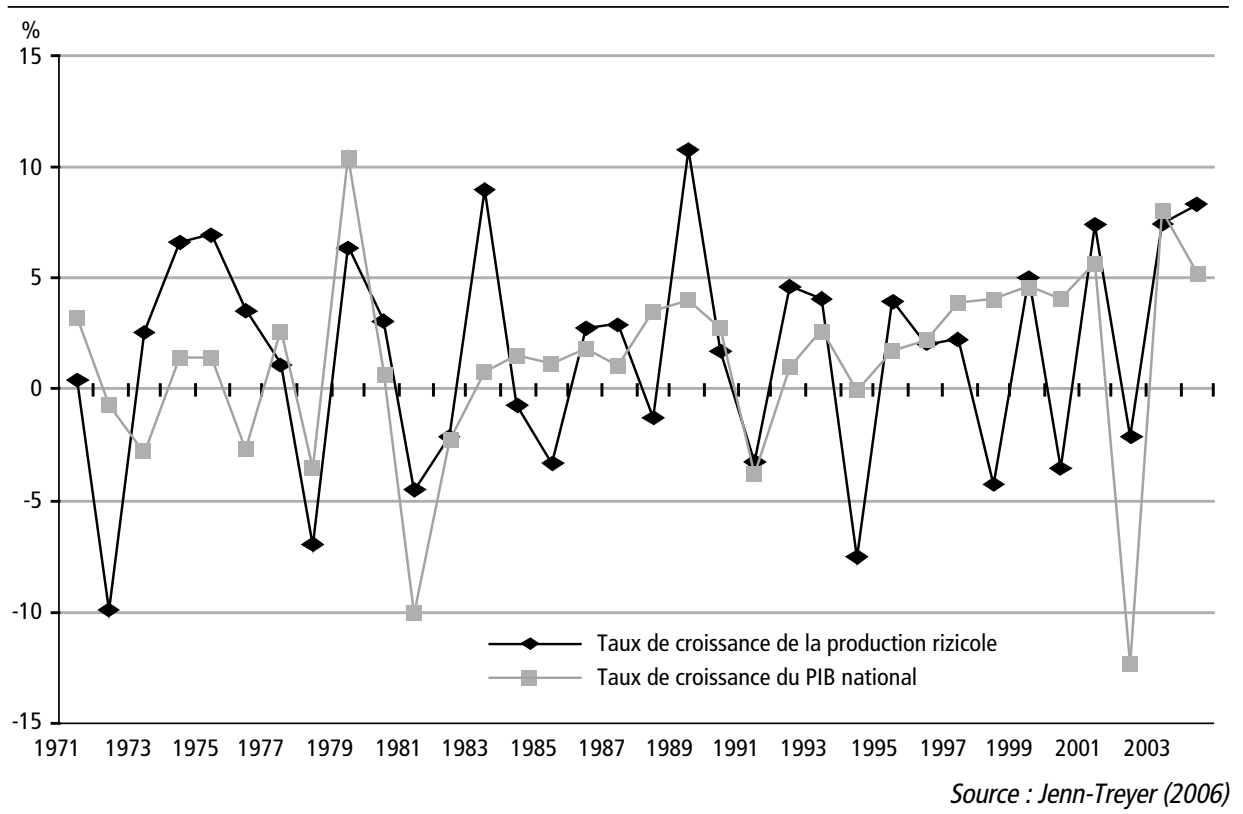

la sécurité alimentaire, la lutte contre la pauvreté et la préservation de l'environnement.

\section{Un moteur de développement économique}

Madagascar est une économie fondée sur le riz comme l'ont montré plusieurs travaux (Le Bourdiec, 1974 ; Roubaud, 1997 ; FOFIFA/ IFPRI, 1997 ; UPDR/FAO/CIRAD, 2000 ; Minten et Zeller, 2000 ; Razafindravonona et al, 2001 ; Bockel, 2002 ; Fraslin, 2002 ; World Bank, 2003). La population agricole constitue près de $80 \%$ de la population active totale et $85 \%$ des exploitants cultivent du riz, sans compter les ménages urbains qui exploitent ou louent leurs propres rizières ; cette céréale est la principale culture en termes d'occupation de la superficie agricole pour les trois quarts des communes malgaches et la principale source de revenu des ménages dans $45 \%$ des communes ${ }^{1}$.

1. Le riz est moins important à l'est du pays où dominent les cultures de rente et dans le sud où pour des raisons climatiques le maïs et le manioc sont plus répandus.
Pour donner quelques ordres de grandeur de la filière, la production nationale de paddy est de 3 millions de tonnes par an et progresse sur le long terme à un rythme inférieur à la croissance démographique. Plus de $60 \%$ de la production est destinée à l'autoconsommation, la filière riz est donc peu intégrée au marché. Les importations varient selon les années et représentent en moyenne $10 \%$ de la production nationale. Enfin, le riz commercialisé, local ou importé, approvisionne à $60 \%$ le milieu urbain (UPDR/FAO/CIRAD, op. cit.).

Les performances de la filière déterminent de manière significative le dynamisme du secteur agricole et de l'économie nationale. Sa valeur ajoutée économique directe, calculée en $1999^{2}$, contribue à hauteur de $12 \%$ au PIB national et de $43 \%$ au PIB agricole en termes courants (Dabat, 2003) ${ }^{3}$.

2. Afin de l'évaluer, la partie autoconsommée du paddy a été valorisée au prix du marché départ producteur.

3. À titre de comparaison, la filière crevette (baptisée «l'or rose » de Madagascar pour ses apports en devises étrangères) représente moins de $1 \%$ du PIB (Henry et al, 1998). 
Une autre façon d'apprécier l'impact dynamique du secteur rizicole sur l'économie nationale est la comparaison du taux de croissance de la production rizicole avec le PIB (graphique 1), révélatrice de la contribution de la filière riz au PIB mais aussi des effets induits par les activités rizicoles sur les autres secteurs de l'économie nationale. Le coefficient de corrélation de Pearson entre les deux séries est égal à 0,486 (significatif à $1 \%$ d'erreur), montrant le rôle moteur de la filière riz dans l'économie nationale.

\section{Le prix du riz, facteur d'équité sociale ?}

Le riz est la principale denrée alimentaire dans la plupart des régions malgaches. Leur sécurité alimentaire est fortement liée à l'abondance des récoltes ainsi qu' au niveau et à la stabilité des prix. Les situations de pénurie, le plus souvent dues à des facteurs naturels (cyclones, fortes pluies, sécheresse...), ont un impact immédiat sur l'approvisionnement des marchés et les rations alimentaires. L'augmentation des prix réduit le pouvoir d'achat des consommateurs nets de riz et diminue leur consommation. A contrario, une baisse des prix touche les vendeurs nets de riz et décourage la production. Les fluctuations saisonnières pénalisent même les ménages autosuffisants en riz qui généralement vendent à la récolte à prix bas et achètent du riz plus cher à la soudure.

Ainsi, les marchés rizicoles jouent un rôle majeur sur les revenus des producteurs (environ $60 \%$ de la population) et le niveau de vie des consommateurs (la population en entier) par le biais des prix. D'où l'intérêt de rechercher une plus grande efficacité du fonctionnement des marchés rizicoles.

\section{L'enjeu écologique}

La demande croissante en riz et l'augmentation de la pression foncière sur les terres inondées liées à la croissance démographique et à la stagnation de la productivité des zones irriguées, conduisent au développement d'une riziculture pluviale sur les collines. Celle-ci présente l'avantage de ne pas exiger d'aménagements coûteux comme c'est le cas pour les cultures irriguées ou l'exploitation des bas-fonds et peut dans certaines conditions offrir des rendements aussi élevés que la riziculture aquatique. Cependant, l'érosion et le ruissellement associés à ce type de culture ont tendance à engendrer la dégradation des sols des collines et à causer des dégâts par ensablement sur les infrastructures en aval.

Le développement de solutions adaptées aux conditions agroclimatiques et socioéconomiques locales qui soient économiquement rentables et facilement applicables, tout en préservant l'environnement, paraît donc un enjeu capital pour le pays. Les techniques agro-écologiques de « semis direct sur couverture végétale permanente » ou SCV peuvent contribuer à relever ce défi (Chabierski et al, 2005). Madagascar a fait le choix d'introduire récemment ces techniques au sein des systèmes culturaux. Les résultats de la diffusion de ces systèmes dépendent cependant de plusieurs facteurs : la mise au point de variétés et techniques culturales diversifiées et appropriables par les communautés paysannes ainsi que l'existence d'un environnement économique favorable à leur adoption, facilitant notamment l'accès aux intrants, au crédit et aux marchés.

\section{La faible performance économique de la filière riz}

Plusieurs indicateurs physiques et économiques révèlent la faible performance de la filière riz malgache qui se caractérise par une difficulté à accumuler le capital et une compétitivité limitée.

\section{Des indicateurs au plus bas}

La riziculture malgache est peu performante. L'accumulation du capital dans la filière se limitait depuis la libéralisation à quelques riziers et grossistes, largement concurrencés aujourd'hui par de plus petits opérateurs, souvent informels, capables de 
réagir rapidement et avec plus d'efficacité (petite taille, localisation près de lieux de consommation, bien informés des évolutions du marché, travaillant en flux tendus...). Concernant les exploitations, en dehors de quelques greniers à riz, la majorité d'entre-elles sont de petite taille, investissent peu, ont un accès limité au crédit, adoptent modérément les innovations techniques, sont peu insérées au marché et poursuivent des objectifs d'autosubsistance.

Le rendement officiel stagne en moyenne à $2 \mathrm{t} /$ ha malgré des disparités importantes selon les systèmes de production et les régions. La comparaison avec d'autres pays où le riz est la culture vivrière principale, met en évidence la faiblesse des résultats malgaches. Alors que les rendements en Indonésie et à Madagascar étaient comparables au début des années 1970, l'écart entre les deux pays est actuellement de l'ordre de 2,5 t/ha. De la même façon, les rendements au Mali ont actuellement dépassé les rendements malgaches alors qu'ils étaient à moins de $1 \mathrm{t} / \mathrm{ha}$ au début de cette même période.

Outre le rendement, d'autres indicateurs économiques sont aussi peu convaincants : le niveau des prix réels en 2000-2003 est similaire à ceux antérieurs à la libéralisation du marché (années 1980) montrant que la productivité ne s'est pas améliorée entre ces deux périodes ${ }^{4}$ (Minten et al, 2006) ; les fortes variabilités saisonnière et annuelle des prix continuent à avoir un impact dépressif sur le pouvoir d'achat de la population dans l'impossibilité de stocker ${ }^{5}$ (Dorosh et Minten, 2006) ; la disponibilité en riz par habitant est passée de $180 \mathrm{~kg} / \mathrm{hab}$. au début des années 1960 à $100 \mathrm{~kg} / \mathrm{hab}$. à la fin des années 1990 ; la productivité du travail est beaucoup plus faible que dans

4. Contrairement à d'autres pays comme le Bangladesh par exemple.

5. Parmi les ménages malgaches, $46 \%$ sont des acheteurs nets de riz, $19 \%$ seulement sont vendeurs nets et $11 \%$ sont autosuffisants. les pays rizicoles asiatiques (Bockel et Dabat, 2001 ; Bockel, 2003) ; les années récentes se sont caractérisées par des niveaux élevés d'importation de riz (2000, 2001, 2003) qui traduisent le décrochement sur longue période de l'offre par rapport à la demande ; plusieurs diagnostics ont montré en 2004-2005 l'état dégradé de l'outil de production rizicole et notamment des infrastructures hydrauliques (Banque mondiale, AFD, IWMI, BRL...) ; enfin, des études récentes pointent l'importance de la paupérisation rurale et de l'insécurité alimentaire difficiles à éradiquer (FOFIFA, INSTAT, PAM, FAO, CRS, Cornell University.... ${ }^{6}$

\section{Un paradoxe des prix}

Les consommateurs urbains achètent le riz à des prix significativement plus élevés que le cours international alors que les producteurs vendent leur paddy à un prix en dessous des coûts à l'étranger. La nouvelle économie institutionnelle fournit des outils utiles à la compréhension de cette faible compétitivité qui peut s'expliquer par le dysfonctionnement des marchés et l'importance des coûts de transaction (Jouanjean, 2005). Les coûts de transport élevés à l'intérieur et en provenance de l'extérieur du pays, l'insécurité rurale et les vols de produit, l'accès limité au crédit, notamment pour le stockage, et d'autres contraintes au sein de la filière, sont à l'origine de coûts de transaction élevés qui tirent à la baisse les prix au producteur et limitent la commercialisation (Minten et Dabat, 2006).

Ainsi, il n'y a pas de bénéfices au commerce, les investisseurs potentiels limitent les échanges et la spécialisation et réduisent la productivité globale, en détour-

6. AFD : Agence française de développement ; IWMI : International Water Management Institute ; BRL : Bas-Rhône Languedoc ; FOFIFA : Centre national de recherche appliquée au développement rural ; INSTAT : Institut national de la statistique ; PAM : Programme alimentaire mondial ; FAO : Organisation des Nations-Unies pour l'Alimentation et l'Agriculture; CRS : Catholic Relief Services. 
nant les investissements maximisant les rendements vers ceux minimisant le risque, le marché (crédit, intrants, produits...) étant perçu comme défaillant (North, 1990). Les échanges sont caractéristiques d'un « marché aux puces» (Jouanjean, op. cit.) qui permet des transactions ni sur des volumes importants, ni sur des distances longues.

Il y a un manque d'investissements dans les actifs spécifiques nécessaires au développement des systèmes d'offre d'intrants, du financement agricole, de la commercialisation, des transports et des infrastructures d'irrigation. Le secteur privé n'a pas été capable d'apporter les services nécessaires à une augmentation de la production. Malgré l'entrée en activité de nombreux agents dans la filière riz, le marché n'atteint pas pour autant un équilibre concurrentiel. Cette lacune confine les acteurs dans une trappe à pauvreté (Hoff et Beyond RosensteinRodan, 2000).

Les théoriciens préconisent le développement d'un environnement institutionnel et d'arrangements institutionnels « hors marché » (Kydd et Dorward, 2003) pour améliorer la performance de la filière et par extension de l'économie, à travers ses effets sur la limitation des coûts et des risques de transaction et le développement de formes plus complexes d'échanges (North,op. cit.). La création d'institutions facilitant la contractualisation entre acteurs et la résolution des conflits favoriserait une bonne coordination économique des agents, garante du développement de la production rizicole.

\section{Évolution historique des modes de régulation : de l'État au marché}

Le principal point d'ancrage des politiques publiques concernant la filière riz à Madagascar demeure le prix du riz, facteur de performance économique et d'équité sociale. L'État intervient tour à tour pour stabiliser les prix afin de satisfaire les consomma- teurs ou encourager la commercialisation et stimuler les riziculteurs. Cette partie montre l'évolution chaotique des modes de régulation depuis un pilotage étatique unilatéral jusqu'au milieu des années 1980 vers une forte libéralisation de la filière ensuite.

\section{Les années 1960 à 1980 : les déboires de l'intervention de l'État}

À Madagascar, le riz a toujours été considéré par l'État comme un produit capable de mettre en danger sa stabilité politique. Aussi, depuis son indépendance en 1960, et jusqu'à la fin des années 1980 , le pays a connu divers systèmes de régulation étatique du marché du riz se caractérisant principalement par l'appropriation des moyens de production et un contrôle des prix.

\section{La nationalisation de l'aval de la filière}

Dans les années 1960, le secteur rizicole était entre les mains du secteur privé avec une intervention publique minime. Dès 1963, l'État s'immisce dans les activités rizicoles en créant le Bureau de commercialisation et de stabilisation du riz (BCSR). Le contrôle de l'État coexistait au début avec le secteur privé, mais il s'est durci par la suite, avec d'abord, en 1970, l'entrée en vigueur du monopole du BCSR au Lac Alaotra puis, à partir de 1973, avec l'éviction totale du secteur privé de la commercialisation du riz.

De 1973 à 1977, la Société d'intérêt national des produits agricoles (la Sinpa), entreprise parapublique, détient le monopole de la collecte et de la transformation du paddy ainsi que de la commercialisation interne et externe du riz. La Sinpa s'appuyait notamment sur deux sociétés d'aménagement, la Somalac (Société malgache du Lac Alaotra, créée en 1961) et le Comema (Comité d'expansion économique de la plaine de Marovoay, créé en 1964) transformé en une société d'État en 1974 (Fifabe).

Face aux difficultés administratives et techniques de la Sinpa, liées à la gestion d'une structure aussi lourde et inadaptée 
aux réalités des campagnes, et à l'éclatement des bassins de production secondaires, l'État a progressivement étendu le monopole à d'autres entreprises publiques ou parapubliques pour se substituer au réseau très ramifié de collecteurs qui existait précédemment.

\section{Le contrôle étatique du système des prix}

Afin d'assurer la satisfaction des besoins essentiels de la population, notamment des couches défavorisées, l'État fut amené à contrôler les prix sur le marché des biens alimentaires. Cela nécessitait d'importantes subventions, notamment lorsque les denrées alimentaires importées subissaient une forte hausse des prix ou lorsque la production locale était insuffisante. La politique de prix interventionniste et le contrôle des circuits de distribution dans les années 1970 ont donc surtout favorisé le milieu urbain.

Le BCSR avait pour mission de réguler le prix du riz en achetant le paddy aux producteurs par l'intermédiaire de mandataires agréés. L'action de la Sinpa eut pour conséquence de déconnecter les prix au producteur des prix internationaux et des coûts de production.

D'autres éléments que la lourdeur de la structure mise en place, expliquent la déliquescence du système. La fixation du prix du paddy aux producteurs à des niveaux de moins en moins incitatifs (Roubaud, 1997) ou le fait que le riz était payé aux producteurs en bons administratifs (parfois non remboursés par l'État), ont contribué à l'enclenchement d'un processus d'involution rurale (Pesnaud, 1997), c'est-à-dire de repli du monde rural sur lui-même.

La production moyenne officiellement commercialisée est alors passée de 175000 tonnes en 1970-1971 à 155000 tonnes en 1976-1977. Les opérations de commercialisation ont rencontré de sérieux problèmes, dus aux coûts élevés des activités de collecte, de stockage, mais également à l'insuffisance de la production, et par conséquent, à la nécessité d'importer massivement du riz. Ainsi, au début des années 1980, à peine moins de $10 \%$ de la production était collectée par l'État et mise sur le marché. Les importations de riz par l'État pour contrer la pénurie dans les centres urbains sont passées de 2500 tonnes en 1971 à 135000 tonnes en 1977 , représentant $40 \%$ de la production locale et ont culminé à un pic historique de 355000 tonnes en 1982.

\section{Le milieu des années 1980 : des résultats peu perceptibles du schéma libéral}

À partir du début des années 1980, Madagascar, comme d'autres pays d'Afrique sub-saharienne, s'est engagé dans une série de mesures d'ajustement structurel, sous l'impulsion du Fonds monétaire international (FMI) et de la Banque mondiale. Le processus de libéralisation progressive du marché rizicole, s'est déployé jusqu'en 1990. L'État s'est désengagé petit à petit du secteur de l'irrigation, de la distribution des intrants et de la vulgarisation des techniques agricoles ; mais la libéralisation des prix a été le fer de lance de la politique rizicole malgache.

\section{L'incitation par les prix}

Les premières mesures d'ajustement dans le secteur rizicole ont recherché l'incitation à la production de paddy en favorisant, dans un premier temps, l'augmentation des prix au producteur par le biais d'un relèvement forfaitaire ${ }^{7}$ puis, dans un second temps, la libéralisation des prix et des circuits de commercialisation.

La suppression du monopole de la Sinpa à partir de 1983 hors des deux zones greniers à riz, le Lac Alaotra et la plaine de Marovoay, fut étendue à toutes les zones productrices en 1986. À cette date, la SINPA n'assurait plus que les importations. En 1990, les importations sont rendues au secteur privé,

7. Dans le cadre de l'accord avec le FMI, les prix au producteur étaient relevés de $27 \%$ en 1982 et de $11 \%$ en 1985. 
mettant ainsi fin à l'intervention publique dans la commercialisation.

\section{Une libéralisation graduelle et partielle} Pour accompagner le mouvement de libéralisation, des opérations de contrôle des marchés par l'État ont encore eu lieu jusqu'en 1991. En dehors des marchés libres, les autorités ont continué à commercialiser du riz, à des prix largement inférieurs à ceux du marché. Cette opération, connue sous le nom de « riz fokontany », a été mise en place en 1983, et s'est poursuivie jusqu'en octobre 1988. Jusqu'en 1986, ce riz représentait $60 \%$ du riz commercialisé à Antananarivo (Roubaud, op. cit.). De 1986 à 1991, un système de « stock tampon » (riz importé) a fonctionné. Mis en place avec l'appui des bailleurs de fonds (FAO, PAM...), ce stock se voulait être une alternative à l'opération riz fokontany, qui faisait peser sur les finances de l'État une charge insurmontable et était vecteur d'une série d'effets pervers liés à la distorsion des marchés.

Le PAM (1991) montre que le système, conçu comme la constitution d'un stock de riz maintenu pour n'être libéré sur le marché que lorsque les fluctuations poussent le prix au-delà d'un prix d'intervention fixé en début de saison, n'a véritablement fonctionné que durant trois ans. En effet, les interventions de ce stock ont vite davantage obéi à la nécessité de liquider les quantités restantes plutôt qu'au besoin de réguler les prix à la consommation. Les coûts de gestion prohibitifs du stock de régulation par les organismes chargés de leur administration ont accéléré sa suppression en 1990.

L'État a cependant conservé un mode d'intervention indirect sur la filière par le biais des politiques monétaire et fiscale. Les dévaluations successives avant 1987 visaient l'augmentation des prix des biens échangeables afin de relancer et de renforcer les substitutions aux importations. Malgré des épisodes de déprotection dus notamment à un souci de préserver la stabilité des prix locaux en période de soudure pendant les années à fort déficit, la taxation des importations reflétait la volonté de protéger la production locale. Fixée à $30 \%$ au début des années 90, la taxe passa à $10 \%$ en 1996 , puis à $30 \%, 10 \%$ et $5 \%$ en 1999 . La loi de finances 2000 a porté les tarifs d'importation à $35 \%$. Une suppression de la taxe a été votée en juillet 2005 , face à la cherté du riz sur le marché international.

\section{L'impact limité de la libéralisation}

Conformément à l'analyse néo-institutionnelle, la libéralisation économique n'a pas sorti les acteurs de la filière riz de la trappe de pauvreté. La politique des prix et le désengagement de l'État ont eu des résultats limités car les conditions d'une relance de la riziculture n'étaient pas réellement en place (Hirsch et Leenhardt, 1993). La réponse de l'offre aux prix a été décevante et insuffisante pour un retour à une croissance soutenue du fait de l'enclavement des régions productrices et de la structure non concurrentielle de la commercialisation (difficultés d'approvisionnement en intrants, coûts importants d'évacuation des produits), « les signaux de prix » atteignant difficilement les producteurs (Razafimandimby, 1998). L'accès des producteurs à un marché local reste une contrainte.

Les travaux de FOFIFA/IFPRI (1997) montrent qu'entre 1992 et 1997, on est passé de $45 \%$ à seulement $51 \%$ des ménages agricoles ayant un accès permanent à plusieurs commerçants pour vendre leurs produits, laissant dans $27 \%$ des villages une situation de quasi-monopole permanent d'un seul commerçant et dans $22 \%$ une situation de quasi-monopole occasionnel.

D'après Roubaud (2000), s'il est à peu près universellement admis que l'économie de marché constitue un puissant moteur de développement, elle ne peut exercer pleinement ses effets qu'associés à un État fort, ne pas

8. TVA : $20 \%$; Tarif d'importation : $15 \%$. 
entendre autoritaire. De l'avoir trop longtemps négligé, pour des raisons à la fois historiques (échec des stratégies de développement fondées sur l'interventionnisme de l'État) et idéologiques (application sans nuance du paradigme néolibéral), les politiques d'ajustement n'ont pas eu les effets escomptés.

\section{La crise 2004 : du marché à l'État}

La période visitée se termine par une « crise fondatrice » récente sur le marché du riz ${ }^{9}$, qui a pointé une intervention peu efficace de l'État car créatrice de comportements d'attentisme et de repli des agents de la filière et la nécessité d'un rééquilibrage au final des rôles dévolus à l'État et au marché. L'originalité de cette crise tient au fait qu'elle semble avoir fonctionné comme un révélateur des véritables mécanismes qui régissent le fonctionnement du marché du riz et fait prendre conscience à l'ensemble des acteurs (État, consommateurs, producteurs) que l'état du marché national dépend beaucoup moins du niveau de l'offre nationale que de la situation du marché international.

\section{Les causes de la crise}

La manifestation la plus visible de la situation de crise du marché du riz en 2004 et début 2005 a été une augmentation substantielle des prix de détail, due à une combinaison de facteurs physiques et monétaires, externes et internes : un renchérissement important du riz sur le marché international ; simultanément, des intempéries cycloniques dans le

\footnotetext{
9. Rappelons que cette crise est « fondatrice » dans le sens où elle pourrait bien déclencher des gains de productivité et une accumulation du capital jusqu'ici insuffisante dans la filière.

10. L'Ariary a connu une dépréciation à partir de décembre 2003 (1 476 Ar pour $1 €$ ), sa parité la plus faible a été atteinte en juin 2004 (2 777 Ar) et s'est stabilisée ensuite (autour de $2500 \mathrm{Ar}$ ) renforçant de fait la compétitivité du riz malgache sur le marché extérieur aux cours plutôt soutenus mais renchérissant encore les importations.
}

pays qui ont tendu le marché en absorbant une partie des stocks nationaux ; et une dépréciation brutale de la monnaie malgache ${ }^{10}$ (Dabat et al, 2006). La crise a atteint son paroxysme à partir d'octobre en raison d'un manque physique de riz sur les marchés. Cette pénurie a généré une flambée des prix du riz local, largement au-dessus de leur niveau de parité avec le prix du riz importé (Jenn-Treyer et Magnay, 2005).

Le jeu naturel des forces de marché et la très forte substituabilité du riz local et du riz importé impliquent que les prix de ces deux types de riz ont tendance à être très fortement corrélés (graphique 2). Et, naturellement, c'est le prix du riz sur le marché national qui, en temps normal, s'aligne sur le prix du riz international. Le suivi des prix fait apparaître, dès le mois d'avril, une rapide augmentation du prix d'importation (prix de vente libre du riz international sur le marché national), par rapport au prix du riz national et du riz importé (vente du riz précédemment importé et dont le prix n'est pas relié au prix d'importation courant), qui restent relativement stables.

Les décisions d'importation sont prises par les opérateurs sur la base d'un arbitrage entre le prix de détail du riz local et le prix d'importation du riz. En termes économiques, un opérateur début 2004 avait certainement plutôt intérêt à acheter son riz sur le marché de gros local plutôt que de l'importer à un prix supérieur. Cet arbitrage des négociants en faveur du riz national a certainement été un des facteurs déterminants de la crise d'approvisionnement du marché du riz. Ce choix des importateurs privés a été renforcé par la triple incertitude dans laquelle ils se trouvaient: incertitude sur la durée et l'ampleur de la dépréciation de la monnaie nationale, sur la nature de la réaction gouvernementale, sur les variations du prix mondial du riz.

Face à cet arbitrage des importateurs largement en défaveur du riz extérieur les premiers mois de 2004, l'État a diligenté une opération d'importation avec quelques opérateurs privilégiés et mis en place une politique des prix 
RECHERCHES

Graphique 2. Prix riz local et importé constatés et prix d'importation calculé riz Viet ( $25 \%$ de brisures Antananarivo)

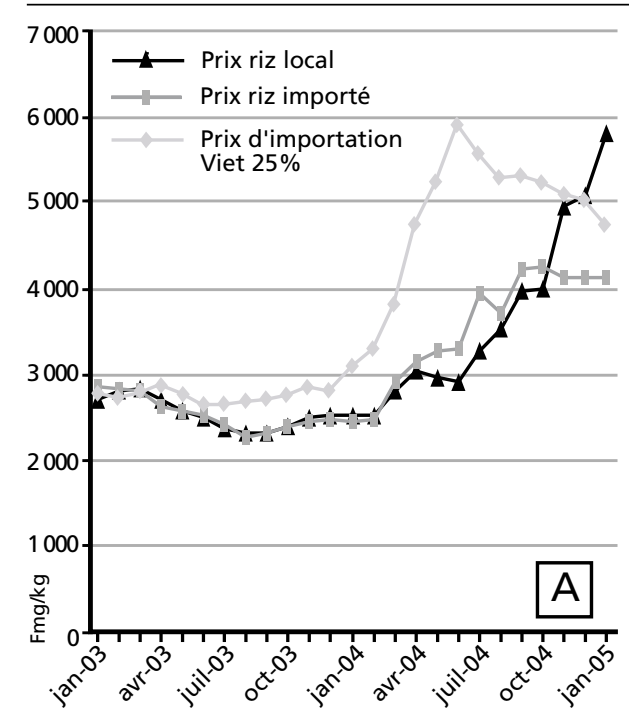

en subventionnant de fait l'importation de riz et administrant son prix de vente (3 500 francs malgaches, Fmg ou 700 Ariary, Ar) largement en dessous du prix d'importation. Cependant ce « riz gouvernemental » est arrivé tard et s'est avéré insuffisant, créant une situation de pénurie généralisée et la flambée des prix intérieurs.

\section{L'État aurait-il pu gérer autrement la crise ?}

La situation paraissait évitable. Si l'ensemble des taxes (y compris la TVA) avait exceptionnellement été suspendu dès juin 2004 ; à partir d'août 2004 les arbitrages des opérateurs seraient devenus favorables au choix du riz importé. Cette allégation correspond, sur le graphique, au passage de la courbe gris clair en-dessous de la courbe noire (graphique 2, passage des situations A à B).

Ainsi, la politique de l'État, à visée sociale (contenir le prix du riz sur les marchés urbains), a créé des distorsions économiques sur le marché du riz. Elle a eu également des effets pervers au niveau social. De manière générale, les agents de la filière qui ont bénéficié de la situation en

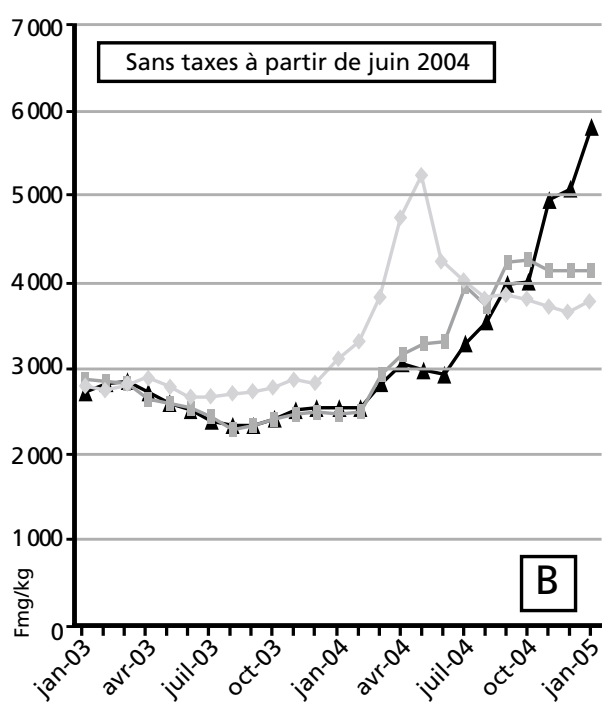

Source : Jenn-Treyer et Magnay, 2005.

2004 sont les riziculteurs excédentaires au cycle tardif et les agents qui ont correctement anticipé l'augmentation des prix et pu mobiliser des capacités et des moyens financiers pour le stockage, les ménages pauvres d'Antananarivo qui ont momentanément acheté du riz subventionné. En 2004, la majorité des riziculteurs n'auraient pas bénéficié de l'augmentation des prix puisqu'ils ont vendu leur paddy à un prix semblable à 2003 à la récolte et ont acheté du riz à un prix très élevé à la soudure (Dabat et al, 2006). En 2005, l'ensemble des riziculteurs ont bénéficié de l'amélioration du prix du paddy.

On peut penser a posteriori qu'en août 2004, une position politique extrêmement claire, fruit d'une concertation entre l'ensemble des acteurs de la filière, associant l'engagement de l'État de ne pas intervenir sur le marché et la suspension transitoire et exceptionnelle des taxes, aurait permis d'atténuer la crise, en stabilisant le prix du riz sur les marchés un peu en dessous de 4000 $\mathrm{Fmg} / \mathrm{kg}$ (sans taxes), soit $800 \mathrm{Ar} / \mathrm{kg}$. De manière moins radicale, la TVA aurait pu être supprimée, et la taxe d'importation 
ramenée à 10-15\%, ce qui aurait stabilisé le prix sur le marché du riz aux alentours de $4500 \mathrm{Fmg} / \mathrm{kg}(900 \mathrm{Ar} / \mathrm{kg}$ ). Cette fourchette de prix est assez proche du prix de parité du riz importé.

\section{Perspectives d'avenir : quel mode de régulation idoine pour la filière?}

À la faible accumulation structurelle du capital dans la filière riz, s'ajoute ainsi une crise récente qui pourrait bien modifier en profondeur les processus de croissance et de régulation. Cette crise a entraîné le retour malhabile de l'État dans la filière riz, imposant de fait des restrictions aux importations de riz, réglementant les prix et réinvestissant la distribution directe et les ventes de riz.

Au début de l'année 2005, la situation ressemblait étrangement à la période qui avait vu le passage de l'opération riz fokontany à la mise en place d'un stock tampon à la fin des années 1980. L'opération « riz importé à $700 \mathrm{Ar}$ » ne pouvant durer trop longtemps du fait de son coût et des effets pervers qu'elle générait, l'État envisageait de la remplacer par un système de stock tampon. Les mêmes solutions risquant de produire les mêmes effets, le besoin de règles du jeu innovantes et de nouveaux outils de régulation qui pourraient s'appuyer plus sur les mécanismes du marché, s'est fait sentir.

Plus exactement, c'est la variation relative des prix intérieurs du riz par rapport au prix international, par les dysfonctionnements du marché domestique qu'elle a entraînée, qui est à l'origine de la recherche de nouveaux compromis institutionnalisés, sur la base d'une compréhension des mécanismes qui ont sous-tendu la crise. Après le « tout État » des années 1970 et le retour à un système plus libéral des années 1980 et 1990, l'émergence de la possibilité d'une gestion alternative, révélée par la crise de 2004-2005, autorise à penser qu'un nouveau cadre de régulation est envisageable, en conformité avec la théorie économique et avec les bailleurs de fonds et les agences de coopération $^{11}$.

\section{Une redéfinition du rôle de l'État}

Mettre l'État au service du marché ne dispense pas, bien au contraire, d'une intervention publique confortée en matière d'investissement et dans certains domaines pour accroître la productivité rizicole et diminuer les coûts de transaction liés à la commercialisation du riz : investissements en irrigation, mise à disposition d'engrais de qualité, construction d'infrastructures routières, amélioration de l'accès des riziculteurs au crédit, diminution de l'insécurité rurale, appui à l'organisation et à la coordination des acteurs, réduction des asymétries d'information en facilitant la circulation des données sur les prix et les marchés.

En revanche, en matière de politique des prix, les mesures indirectes telles que les incitations fiscales paraissent plus appropriées que les mesures directes d'intervention sur le marché, dans la mesure où elles sont à la fois moins coûteuses et moins génératrices de distorsions. L'option «stocks de sécurité » renchérirait considérablement le prix du riz alors que les pénuries liées à une mauvaise récolte sont généralement peu importantes et peu fréquentes. A contrario, des simulations récentes (Dorosh et Minten, op. cit. ; Jenn-Treyer et Magnay, 2005) ont révélé l'efficacité d'une réduction de la taxe à l'importation pour stabiliser les prix du riz. Ces analyses montrent l'intérêt de ne pas manipuler trop fréquemment cette taxe, d'une part pour améliorer la lisibilité de la politique fiscale ; et d'autre part pour éviter une trop forte

11. L'autonomie de décision de l'État malgache en matière d'élaboration de politique agricole s'est accrue depuis quelques années (injonction vaine des bailleurs pour une réduction de la taxe d'importation au moment de la crise, remplacement du Document stratégique de réduction de la pauvreté par le Madagascar Action Plan, diversification de l'aide...). La concertation des bailleurs de fond appuie la stratégie définie par l'État malgache. 
pression sur le gouvernement pour baisser les taxes dans les autres secteurs d'importation. L'option « libéralisation du marché » (pas de stocks publics, pas de restrictions aux importations privées, révision annuelle de la taxe à l'importation) permet d'atténuer les effets d'une variation importante des prix sur le marché international. Les pertes fiscales liées à la diminution des taxes à l'importation sont minimes comparées au coût d'une politique publique de stockage et sont partiellement compensées par l'augmentation du volume importé.

La question paraît ne plus être de choisir entre «plus d'État» ou «plus de marché » mais d'opter plutôt pour «mieux d'État » et un marché plus efficace et équitable. SalazarXirinachs (1993) définit à bon escient la fonction économique centrale de l'État comme étant celle qui se donne comme objectif d'élaborer une vision stratégique du processus de développement, de maintenir les équilibres macroéconomiques et un climat d'investissement approprié ; et d'engager, de manière constructive, moyennant le dialogue et la concertation, tous les acteurs sociaux et politiques dans cette stratégie. Ainsi, il s'agit pour l'État de réunir les conditions favorables au fonctionnement d'un marché libre et en voie d'organisation.

\section{Des règles du jeu claires et connues de tous}

La restauration de la confiance et l'amélioration de la communication entre le secteur privé et les services gouvernementaux, et entre les acteurs eux-mêmes du secteur privé pour faciliter la circulation du produit, apparaissent cruciales. Les marchés rizicoles à Madagascar se caractérisent par une faible confiance entre agents (spécialement l'État et le secteur privé). Les commerçants sont souvent qualifiés de «spéculateurs », les coûts et les risques qu'ils assument et les services qu'ils rendent (notamment le transfert des surplus saisonniers vers la période de soudure) sont souvent sous-estimés. Ce manque de confiance conduit à une insuffi- sance de transparence et à une grande volatilité des prix.

Les objectifs de l'État en matière de régulation du marché doivent être clairs : réduire les interventions gouvernementales directes et aléatoires, de façon à accroître la prédictibilité et l'efficacité des marchés (JennTreyer et Magnay, op. cit.). Ceci suppose, par exemple, que l'État fixe le niveau des taxes à l'importation au début de l'année juste avant la récolte, qu'elles ne varient pas en cours d'année et que ces taxes soient les mêmes pour tous les importateurs. Le développement de politiques transparentes tenant compte des avis de tous les groupes d'acteurs doit être prôné.

\section{La plateforme de concertation : outil de compromis institutionnalisé ?}

La filière riz malgache se trouve dans une trappe à pauvreté du fait de la carence d'investissements, de l'étroitesse du marché et de la faiblesse des institutions facilitant la coordination économique entre les agents. L'innovation institutionnelle et la coordination hors-marché sont préconisées par la théorie économique pour permettre l'investissement dans les actifs spécifiques et sortir des trappes à pauvreté.

Dans les économies coordonnées, existent des mécanismes de délibération collective et de mise en place d'institutions pour la promotion et la mise en œuvre d'actions coordonnées et une compréhension partagée des objectifs et des conséquences distributives de telles actions. L'État peut faciliter ces processus délibératifs et de coordination et aider à appliquer les stratégies qui en sont issues. Cependant bien que l'implication de l'État soit nécessaire pour guider la coordination hors-marché, une implication trop forte peut aussi être problématique à cause de l'imperfection de l'information, des changements d'objectifs et de la réserve des agents face à la possibilité de changement unilatéral des règles du jeu par le gouvernement. L'État devrait voir sa responsabilité limitée à l'encouragement de la 
coordination comme n'importe quel autre bien public, les acteurs de la filière étant chargés de la promouvoir à travers des associations de producteurs et de commerçants notamment. Idéalement la coordination est basée sur des institutions délibératives travaillant horizontalement dans un soussecteur mais aussi verticalement dans la filière.

Le contexte de crise qui prévaut dans la filière riz à Madagascar constitue une période opportune pour la mise en place d'une forme d'organisation créant des règles, des droits et des obligations pour les parties prenantes qui s'imposeraient à tous et dont découleraient des comportements et des stratégies plus efficaces des agents de la filière. En particulier, l'établissement de nouvelles règles d'intervention des acteurs nous paraît devoir permettre l'élaboration de compromis institutionnalisés portant sur le pilotage de la filière et donner sens au partenariat public privé. La Plateforme de concertation et de pilotage de la filière riz (PCPRIZ), en cours de construction, pourrait devenir l'outil de cette coordination.

Depuis 2002, l'État (ministère de l'Agriculture, de l'Élevage et de la Pêche ; ministère du Commerce et de la Consommation), les bailleurs de fonds, les organismes de coopération et les opérateurs tentaient de mettre en place une structure de réflexion, de concertation, de pilotage et de suivi d'un programme d'actions pour la filière, sans beaucoup de succès en matière d'opérationnalité. Au cours de la période de sortie de crise, durant laquelle d'intenses discussions ont permis de reconsidérer les positions en place, le lancement effectif d'un pilotage mixte de la filière, associant à la prise de décision des acteurs de plusieurs natures et notamment publics et privés, a vu le jour.

Cette structure est au carrefour de plusieurs missions : favoriser en toute transparence les dialogues, les rencontres, la collecte et les échanges d'informations entre les acteurs directs et indirects de la filière, orienter les actions nécessaires en vue de proposer une politique de développement de la filière, tenir un rôle d'interface entre les producteurs et l'État, discuter des mesures, suivre la mise en œuvre de la politique du sous-secteur rizicole à Madagascar : la refonte de la politique semencière, la politique en matière d'engrais, le développement d'un système de conseil agricole, l'initiation des exportations et la création d'un système d'informations de marché.

Notons qu'une des premières actions significatives de la PCPRIZ a été de conduire le dialogue entre importateurs de riz, à partir du mois d'octobre 2005, afin que ceux-ci revoient à la baisse leurs niveaux d'importation, qui auraient sans cela été beaucoup trop importantes, risquant alors de créer un accident majeur sur le marché et de léser les petits producteurs stockeurs. Signalons par ailleurs que cette décision concertée a été prise contre l'avis du gouvernement, qui préconisait quant à lui des importations les plus massives possibles. Il est donc prématuré de parler de compromis institutionnalisé, par contre cette action a vraiment eu un effet régulateur sur le marché puisque suite à une bonne récolte en 2005 les prix ne se sont pas effondrés en fin d'année comme ils étaient supposé le faire avec des importations incontrôlées.

\section{Des opportunités ouvertes par la crise}

Enfin, la crise a ouvert plusieurs autres opportunités pouvant donner corps et constituer autant de défis à relever par la plateforme pour relancer la filière riz :

- l'augmentation du prix du paddy si celuici se stabilise à un niveau plus élevé qu'auparavant offre aux paysans excédentaires la possibilité de cibler le riz comme culture commerciale et pas seulement vivrière ${ }^{12}$;

12. Mais les riziculteurs pourraient bien aussi être incités à produire plus à l'avenir pour limiter les risques de pénurie pour les besoins de leurs ménages en mettant en culture toutes les terres exploitables. 
- le ratio prix de l'engrais/prix du paddy s'est amélioré pouvant inciter les riziculteurs à utiliser plus d'engrais ${ }^{13}$;

- la dévaluation de la monnaie malgache et les cours soutenus du riz au niveau international devraient permettre au riz malgache de redevenir compétitif à l'exportation, surtout vers les destinations du Marché commun d'Afrique orientale et australe (COMESA) et de la Communauté pour le développement de l'Afrique australe (SADC) : le pays pourrait exporter du riz de qualité à prix élevé et continuer à importer du riz ordinaire bon marché, exporter en période de récolte et importer en période de soudure pour réguler les flux de riz et limiter les stocks ;

- la situation 2004 a montré enfin l'importance d'exploiter plus à l'avenir la diversité des possibilités techniques : des cultures marginales, comme le riz précoce (vary aloha, riz pluvial...) ou tardif (vary jeby), ont atténué la pénurie à des moments critiques.

\section{Conclusion}

La filière riz à Madagascar constitue un véritable enjeu de développement durable, pourtant ses faibles performances économiques confinent la plupart de ses agents dans une trappe de pauvreté. Cet état de fait s'est aggravé avec la crise du marché du riz de 2004-2005 qui illustre bien le risque de déstabilisation lié à la dépendance vis-à-vis d'un marché mondial où les hausses de prix internationaux sont accentuées en cas de dégradation du taux de change.

Le diagnostic historique remet en question deux principaux modes de régulation de la filière depuis l'Indépendance, éprouvés sans beaucoup de réussite : un interventionnisme généralisé de l'État qui bridait le marché et une libéralisation imposée de la production et des prix sans

13. Du fait de l'augmentation des prix du paddy, le ratio est actuellement à $2 \mathrm{~kg}$ de paddy pour $1 \mathrm{~kg}$ d'engrais mais il est probable que la hausse du prix du pétrole engendrera une forte hausse des engrais sur le marché mondial pour les années à venir. maturité du marché. Chacun de ces modes de régulation a été sanctionné par un affaiblissement de la production intérieure et un appel important aux importations (plus de 350000 tonnes en 1982, plus de 200000 tonnes en 2000).

Dans un contexte de paradoxe des prix, significatif de la défaillance des marchés, de nouvelles formes institutionnelles pourraient favoriser l'efficacité des processus de production et de commercialisation et d'intégration des marchés. La crise a été le catalyseur qui a permis l'abandon d'une double illusion : celle de l'État qui voyait dans le retour des bonnes recettes centralisatrices et dirigistes une réponse adéquate, et celle du marché et de ses animateurs, pour qui la liberté absolue (sans règles) était la panacée. Elle a ouvert la voie à la mise en place de nouvelles règles et de nouveaux outils et a élargi le spectre des perspectives d'avenir pour la filière.

Un nouveau cadre de régulation pourrait limiter l'intervention de l'État sur le marché à la création des conditions favorables au fonctionnement d'un marché libre et en voie d'organisation et réhabiliter à cette fin la mise en place de politiques publiques fortes et affirmées pour faciliter les investissements privés dans les actifs spécifiques (intrants, crédit...) et la sécurisation de l'ensemble de l'environnement économique des agents de la filière.

L'enjeu est donc double, à la fois la sortie de crise et le positionnement sur une trajectoire d'accumulation réelle et durable, en cela cette rupture peut être qualifiée de crise fondatrice. La façon dont la coordination des acteurs parviendra à institutionnaliser des compromis clairs, notamment entre producteurs et consommateurs par le biais des prix, facteurs d'équité sociale et de performance économique, sera un révélateur de l'opérationnalité du nouveau mode de régulation. L'histoire chaotique de la régulation du marché du riz pour une croissance durable de la production rizicole malgache est en marche. 


\section{RÉFÉRENCES BIBLIOGRAPHIQUES}

Bockel L., Dabat M.-H. (2001). Améliorer la productivité du travail dans la riziculture pour lutter contre la pauvreté à Madagascar. Communication au séminaire «La pauvreté à Madagascar : état des lieux, réflexions sur les politiques de réduction et leur mise en ceuvre », Antananarivo, 5-7 février, $12 \mathrm{p}$.

Bockel L. (2002). Review of Madagascar's rice sub-sector. World Bank, Background Report, Madagascar Rural and Environmental Review.

Bockel L. (2003). Filière riz et pauvreté rurale à Madagascar: déterminants d'une stratégie de réduction de la pauvreté rurale et politiques publiques de développement. Thèse de Doctorat, Université de Metz, octobre.

Le Bourdiec F. (1974). Hommes et paysages du riz à Madagascar. Antananarivo, FTM.

Chabierski S., Dabat M.-H., Grandjean P., Ravalitera A., Andriamalala H. (2005). Une approche socio-éco-territoriale en appui à la diffusion des techniques agroécologiques au Lac Alaotra, Madagascar. III $^{\mathrm{e}}$ World Congress on Conservation Agriculture, «Linking Production, Livelihoods and Conservation », Nairobi, Kenya, 3-7 october, 8 p.

Dabat M.-H., Razafimandimby S., Bouteau B. (2004). Atouts et perspectives de la riziculture péri-urbaine à Antananarivo, Madagascar. Cahiers d'études et de recherches francophones Agricultures, vol.13, n¹, p. 99-109.

Dabat M.-H. (2003). Analyse de la filière riz à Madagascar. Memento de l'agronome, CIRAD-GRET-ministère des Affaires étrangères, $15 \mathrm{p}$.

Dabat M.-H., Jenn-Treyer O., Magnay J., Minten B. (2006). The 2004 rice crisis in Madagascar. In Minten B. et Dorosh P. (Eds), « Madagascar in Disarray: Policy Options for Increased Efficiency and
Price Stabilization », World Bank, Africa Region Working Paper, series $n^{\circ} 101$, september, p. 10-15.

Dorosh P., Minten B. (2006). Price stabilization options. In Minten B. et Dorosh P. (Eds), « Madagascar in Disarray : Policy Options for Increased Efficiency and Price Stabilization », World Bank, Africa Region Working Paper, series $n^{\circ} 101$, September, p. 17-21.

FOFIFA/IFPRI (1997). Le marché des intrants, le marchés des produits agricoles, le comportement des ménages ruraux. Octobre, $29 \mathrm{p}$.

Fraslin J.-H. (2002). Quel avenir pour les paysans de Madagascar? Afrique contemporaine, $\mathrm{n}^{\circ}$ 202-203, p. 93-110.

Henry F., Dabat M.-H., Rakotomanjaka J. (1998). La valeur économique de la ressource crevettière à Madagascar. In Kourkouliotis K. et Ranaivoson E. (Eds), «Aménagement de la pêche crevettière malgache », rapport technique de l'atelier, Programme sectoriel Pêche, PNUD/FAO, Antananarivo 17-19 juin, p. 127-160. Hirsch R., Leenhardt B. (1993). Le riz et le problème vivrier malgache. Rapport pour l'AFD, Antananarivo, 75 p. + annexes. Hoff K., Beyond Rosenstein-Rodan (2000). The Modern Theory of Underdevelopment Traps. World Bank Economic Conference.

Jenn-Treyer O., Magnay J. (2005). Mise en place d'un système de régulation sur le marché du riz à Madagascar. Rapport de mission, Programme alimentaire mondial, mai, $66 \mathrm{p}$.

Jenn-Treyer O., Magnay J. (2006). Development of rice markets. In Minten B. et Dorosh P. (Eds), «Madagascar in Disarray: Policy Options for Increased Efficiency and Price Stabilization », World Bank, Africa Region Working Paper, series $n^{\circ} 101$, september, p. 16-17. 
Jouanjean M.-A. (2005). Les apports de la nouvelle économie institutionnelle à l'analyse de la filière rizicole malgache. Mémoire présenté pour le DESS Analyse économique du développement, Université Paris X, 87 p. + annexes.

Kydd J., Dorward A. (2003). Implications of markets and coordination failures for rural development in least developed countries, Development Studies Association Annual Conference, Strath clyde University, Glasgow, 10-12 September, 12 p.

Minten B., Zeller M. (2000). Beyond Market Liberalization: Welfare, Income Generation and Environmental Sustainability in Rural Madagascar. Aldershot, Ashgate.

Minten B., Dabat M.-H. (2006). The rice economy of Madagascar. In Minten B. et Dorosh P. (Eds), «Madagascar in Disarray : Policy Options for Increased Efficiency and Price Stabilization », World Bank, Africa Region Working Paper, series $n^{\circ} 101$, september, p. 1-8.

North D. (1990). Institutions, Institutional Change and Economic Performance. Cambridge, Cambridge University Press.

PAM (1991). Mission de revue des aspects de gestion du projet Madagascar 3123 "Assistance à une opération d'ajustement du secteur agricole - stock régulateur ». Rapport de mission, 14-28 janvier.

Pesnaud F. (1997). La riziculture des Hautes Terres de Madagascar : les performances bridées d'un système de production. In INSTAT, Revue Économie de Mada- gascar, «Agriculture : enjeux et contraintes de la libéralisation », $\mathrm{n}^{\circ} 2$, octobre, p. 89-111.

Razafindravonona J.-D., Stifel D., Paternostro S. (2001). Dynamique de la pauvreté : 1993-1999, Antananarivo, INSTAT.

Razafimandimby L. (1998). L'ajustement structurel dans le secteur agricole de Madagascar : insuffisance des réformes de prix et faiblesse de la compétitivité. Centre d'Économie du Développement de l'Université Montesquieu Bordeaux IV, $14 \mathrm{p}$.

Roubaud F. (1997). La question rizicole à Madagascar : les résultats d'une décennie de libéralisation. In INSTAT, Revue Économie de Madagascar, «Agriculture : enjeux et contraintes de la libéralisation », $\mathrm{n}^{\circ} 2$, octobre, p. 37-61.

Roubaud F. (2000). Identités et transition démocratique : l'exception Malgache? Paris, L'Harmattan.

Salazar-Xirinachs J.-M. (1993). The role of the State and the Market in Economic Development. In Sunkel O. (Eds), «Development from within. Toward a Neostructuralist Approach for Latin America », (chap 13), Boulder, Lynne Rienner Publishers.

UPDR/FAO/CIRAD (2000). Diagnostic et perspectives de la filière riz à Madagascar. Antananarivo, $89 \mathrm{p}$.

World Bank (2003). Reaching the Rural Poor - A Renewed Strategy for Rural Development. Washington D.C. 